\title{
ON THE TODA AND KAC-VAN MOERBEKE HIERARCHIES
}

\author{
GERALD TESCHL
}

\begin{abstract}
We provide a comprehensive treatment of the single and double commutation method as a tool for constructing soliton solutions of the Toda and Kac-van Moerbeke hierarchy on arbitrary background. In addition, we present a novel construction based on the single commutation method. As an illustration we compute the $N$-soliton solution of the Toda and Kac-van Moerbeke hierarchy.
\end{abstract}

\section{INTRODUCTION}

In 1968 Peter Lax [10] presented his famous approach for completely integrable nonlinear evolution equations by rewriting such equations as linear evolution equations for linear operators, viz.

$$
\frac{d}{d t} H(t)=[P(t), H(t)]
$$

where $[P, H]=P H-H P$ denotes the usual commutator. Under suitable conditions, (1.1) will imply existence of a unitary propagator $U(s, t)$ for $H(t)$, that is,

$$
H(t)=U(t, s) H(s) U(s, t), \quad U(t, s)^{*}=U(t, s)^{-1}=U(s, t) .
$$

As a trivial consequence one concludes that the norm $\|H(t)\|$ of $H(t)$ is independent of $t$. And it is this, at first sight innocent looking, fact which provides a uniform bound on the matrix coefficients of $H(t)$ and hence implies uniqueness and (global) existence of solutions for the associated nonlinear evolution equation (see Theorem 2.4 below). It seems like this last fact has not been used in the literature before and that Theorem 2.4 is the first general existence and uniqueness result for bounded solutions of the Toda lattice on the whole line (for the case of the half line see 4, Proposition 1).

The purpose of the present paper is to revisit methods for constructing soliton solutions on arbitrary background and exploit the abovementioned uniqueness and existence result to obtain short and elegant proofs for these methods. In particular, we want to simplify and improve the results of $[8$ and, at the same time, extend all methods to the entire hierarchy.

To set the stage we review some basic facts on the Toda and Kac-van Moerbeke hierarchy in our first two sections. Then we provide a detailed investigation of the system

$$
H(t) u=z u, \quad \frac{d}{d t} u=P(t) u, \quad z \in \mathbb{C}
$$

1991 Mathematics Subject Classification. Primary 58F07, 58F37; Secondary 39A10, 47B39.

Key words and phrases. Toda hierarchy, Kac-van Moerbeke hierarchy, commutation method. Math. Z. 231, 325-344 (1999). 
(in the weak sense) whose solutions are needed in the explicit construction of soliton solutions. This section, in some sense, constitutes the technical heart of this paper.

Our final section will then establish the single and double commutation method as a tool for constructing soliton solutions on arbitrary background for the entire Toda and Kac-van Moerbeke hierarchy. In addition, we will show how the single commutation method can be used in a way which has not been noted in the literature before (Theorem 5.5. As an explicit illustration we compute the $N$-soliton solution of the Toda and Kac-van Moerbeke hierarchy.

\section{The Toda hierarchy}

In this section we introduce the Toda hierarchy using the standard Lax formalism ([10]). We first review some basic facts following [3] and then we prove existence and uniqueness for the initial value problem.

We will only consider bounded solutions and hence require

Hypothesis H.2.1. Suppose $a(t), b(t)$ satisfy

$$
a(t) \in \ell^{\infty}(\mathbb{Z}, \mathbb{R}), \quad b(t) \in \ell^{\infty}(\mathbb{Z}, \mathbb{R}), a(n, t) \neq 0(n, t) \in \mathbb{Z} \times \mathbb{R},
$$

and let $t \mapsto(a(t), b(t))$ be Fréchet differentiable in the Banach space $\ell^{\infty}(\mathbb{Z}) \oplus \ell^{\infty}(\mathbb{Z})$.

Associated with $a(t), b(t)$ is a Jacobi operator

$$
\begin{aligned}
H(t): \ell^{2}(\mathbb{Z}) & \rightarrow \ell^{2}(\mathbb{Z}) \\
f & \mapsto \tau(t) f
\end{aligned},
$$

where

$$
\tau(t) f(n)=a(n, t) f(n+1)+a(n-1, t) f(n-1)+b(n, t) f(n)
$$

and $\ell^{2}(\mathbb{Z})$ denotes the Hilbert space of square summable (complex-valued) sequences over $\mathbb{Z}$. Moreover, choose constants $c_{0}=1, c_{j}, 1 \leq j \leq r, c_{r+1}=0$, set

$$
\begin{aligned}
g_{j}(n, t) & =\sum_{\ell=0}^{j} c_{j-\ell}\left\langle\delta_{n}, H(t)^{\ell} \delta_{n}\right\rangle \\
h_{j}(n, t) & =2 a(n, t) \sum_{\ell=0}^{j} c_{j-\ell}\left\langle\delta_{n+1}, H(t)^{\ell} \delta_{n}\right\rangle+c_{j+1}
\end{aligned}
$$

and consider the Lax operator

$$
P_{2 r+2}(t)=-H(t)^{r+1}+\sum_{j=0}^{r}\left(2 a(t) g_{j}(t) S^{+}-h_{j}(t)\right) H(t)^{r-j}+g_{r+1}(t),
$$

where $S^{ \pm} f(n)=f(n \pm 1)$. Clearly, $(\mathrm{H} 2.1)$ implies Fréchet differentiability of $t \mapsto$ $H(t)$ and $t \mapsto P_{2 r+2}(t)$. Restricting to the two-dimensional nullspace $\operatorname{Ker}(\tau(t)-z)$, $z \in \mathbb{C}$ of $\tau(t)-z($ in $\ell(\mathbb{Z}))$, we have the following representation of $P_{2 r+2}(t)$

$$
\left.P_{2 r+2}(t)\right|_{\operatorname{Ker}(\tau(t)-z)}=2 a(t) G_{r}(z, t) S^{+}-H_{r+1}(z, t),
$$


where $G_{r}(z, n, t)$ and $H_{r+1}(z, n, t)$ are monic polynomials in $z$ of the type

$$
\begin{aligned}
G_{r}(z, n, t) & =\sum_{j=0}^{r} z^{j} g_{r-j}(n, t), \\
H_{r+1}(z, n, t) & =z^{r+1}+\sum_{j=0}^{r} z^{j} h_{r-j}(n, t)-g_{r+1}(n, t) .
\end{aligned}
$$

A straightforward computation shows that the Lax equation

$$
\frac{d}{d t} H(t)-\left[P_{2 r+2}(t), H(t)\right]=0, \quad t \in \mathbb{R}
$$

is equivalent to

$$
\begin{aligned}
& \mathrm{TL}_{r}(a(t), b(t))_{1}=\dot{a}(t)-a(t)\left(g_{r+1}^{+}(t)-g_{r+1}(t)\right)=0, \\
& \mathrm{TL}_{r}(a(t), b(t))_{2}=\dot{b}(t)-\left(h_{r+1}(t)-h_{r+1}^{-}(t)\right)=0,
\end{aligned}
$$

where the dot denotes a derivative with respect to $t$ and $f^{ \pm}(n)=f(n \pm 1)$. Varying $r \in \mathbb{N}_{0}$ yields the Toda hierarchy (TL hierarchy)

$$
\mathrm{TL}_{r}(a, b)=\left(\mathrm{TL}_{r}(a, b)_{1}, \mathrm{TL}_{r}(a, b)_{2}\right)=0, \quad r \in \mathbb{N}_{0} .
$$

Next, we want to make use of the fact that $H(t), \mathrm{i} P_{2 r+2}(t)$ are both bounded and self-adjoint operators. We start with some preliminary definitions.

Let $P(t), t \in \mathbb{R}$, be a family of bounded skew-adjoint operators in some (separable) Hilbert space $\mathfrak{H}$. A two parameter family of operators $U(t, s),(t, s) \in \mathbb{R}^{2}$, is called a unitary propagator for $P(t)$, if

(1) $U(t, s), s, t \in \mathbb{R}^{2}$ is unitary.

(2) $U(t, t)=\mathbb{1}$ for all $t \in \mathbb{R}$.

(3) $U(t, s) U(s, r)=U(t, r)$ for all $(r, s, t) \in \mathbb{R}^{3}$.

(4) The map $t \mapsto U(t, s)$ is Fréchet differentiable in the Banach space $\mathfrak{B}\left(\ell^{2}(\mathbb{Z})\right)$ of bounded linear operators and

$$
\frac{d}{d t} U(t, s)=P(t) U(t, s), \quad(t, s) \in \mathbb{R}^{2} .
$$

With this notation the following well-known theorem from functional analysis holds (essentially [13, Theorem X.69).

Theorem 2.2. Let $P(t), t \in \mathbb{R}$ be a family of bounded skew-adjoint operators such that $t \mapsto P(t)$ is Fréchet differentiable. Then $P(t)$ has a unitary propagator $U(t, s)$ in $\mathfrak{H}$.

Note also $d / d t U(s, t)=-U(s, t) P(t),(t, s) \in \mathbb{R}^{2}$. Applied to our situation this gives another well-known result.

Lemma 2.3. Let $a(t), b(t)$ satisfy $\mathrm{TL}_{r}(a, b)=0$ and (H2.1). Then equation 2.8) implies the existence of a unitary propagator $U_{r}(t, s)$ for $P_{2 r+2}(t)$ such that

$$
H(t)=U_{r}(t, s) H(s) U_{r}(t, s)^{-1}, \quad(t, s) \in \mathbb{R}^{2} .
$$

Thus all operators $H(t), t \in \mathbb{R}$ are unitarily equivalent.

In addition, if $\psi(s) \in \ell^{2}(\mathbb{Z})$ solves $H(s) \psi(s)=z \psi(s)$ then the function

$$
\psi(t)=U_{r}(t, s) \psi(s),
$$


fulfills

$$
H(t) \psi(t)=z \psi(t), \quad \frac{d}{d t} \psi(t)=P_{2 r+2}(t) \psi(t) .
$$

Before we proceed with our investigations of the Toda equations, we want to ensure existence and uniqueness of global solutions. For the half line this has been done in 4, Proposition 1. Unfortunately, this proof cannot be easily adapted to the present setting on the full line. However, since we are using Fréchet rather than weak differentiability here, a much simpler argument works.

We now regard the Toda equations as a flow on the Banach space

$$
M=\ell^{\infty}(\mathbb{Z}) \oplus \ell^{\infty}(\mathbb{Z}) .
$$

Theorem 2.4. Suppose $\left(a_{0}, b_{0}\right) \in M$. Then there exists a unique integral curve $t \mapsto(a(t), b(t))$ in $C^{\infty}(\mathbb{R}, M)$ of the Toda equations, that is, $\mathrm{TL}_{r}(a(t), b(t))=0$, such that $(a(0), b(0))=\left(a_{0}, b_{0}\right)$.

Proof. The Toda equation gives rise to a vector field $X_{r}$ on $M$, that is,

$$
\frac{d}{d t}(a(t), b(t))=X_{r}(a(t), b(t)) \quad \Leftrightarrow \quad \mathrm{TL}_{r}(a(t), b(t))=0 .
$$

Since this vector field has a simple polynomial dependence in $a$ and $b$ it is clearly smooth (i.e., of class $C^{\infty}$ - however, Lipschitz continuous would be sufficient for our purpose). Hence by [1, Theorem 4.1.5 solutions for the initial value problem exist locally and are unique. In addition, by equation (2.13) we have $\|a(t)\|_{\infty}+$ $\|b(t)\|_{\infty} \leq 2\|H(t)\|=2\|H(0)\|$ (at least locally). Thus any integral curve $(a(t), b(t))$ is bounded on finite $t$-intervals and Proposition 4.1.22 of [1] implies global existence.

\section{The KaC-van Moerbeke hierarchy and its Relation to the Toda HIERARCHY}

In this section we review some basic properties of the Kac-van Moerbeke hierarchy and its connection with the Toda hierarchy.

Suppose $\rho(t)$ satisfies

Hypothesis H.3.1. Let

$$
\rho(t) \in \ell^{\infty}(\mathbb{Z}, \mathbb{R}), \quad \rho(n, t) \neq 0,(n, t) \in \mathbb{Z} \times \mathbb{R}
$$

and let $t \mapsto \rho(t)$ be Fréchet differentiable in the Banach space $\ell^{\infty}(\mathbb{Z})$.

Define the "even" and "odd" parts of $\rho(t)$ by

$$
\rho_{e}(n, t)=\rho(2 n, t), \rho_{o}(n, t)=\rho(2 n+1, t), \quad(n, t) \in \mathbb{Z} \times \mathbb{R}
$$

and consider the bounded operators (in $\ell^{2}(\mathbb{Z})$ )

$$
A(t)=\rho_{o}(t) S^{+}+\rho_{e}(t), A(t)^{*}=\rho_{o}^{-}(t) S^{-}+\rho_{e}(t) .
$$

In addition, we set

$$
H_{1}(t)=A(t)^{*} A(t), \quad H_{2}(t)=A(t) A(t)^{*},
$$

with

$$
H_{k}(t)=a_{k}(t) S^{+}+a_{k}^{-}(t) S^{-}+b_{k}(t), \quad k=1,2
$$


and

$$
\begin{aligned}
a_{1}(t) & =\rho_{e}(t) \rho_{o}(t), & b_{1}(t) & =\rho_{e}(t)^{2}+\rho_{o}^{-}(t)^{2}, \\
a_{2}(t) & =\rho_{e}^{+}(t) \rho_{o}(t), & & b_{2}(t)=\rho_{e}(t)^{2}+\rho_{o}(t)^{2} .
\end{aligned}
$$

Now we define operators $D(t), Q_{2 r+2}(t)$ (the Lax pair) in $\ell^{2}\left(\mathbb{Z}, \mathbb{C}^{2}\right)$ as follows,

$$
\begin{aligned}
D(t) & =\left(\begin{array}{cc}
0 & A(t)^{*} \\
A(t) & 0
\end{array}\right), \\
Q_{2 r+2}(t) & =\left(\begin{array}{cc}
P_{1,2 r+2}(t) & 0 \\
0 & P_{2,2 r+2}(t)
\end{array}\right),
\end{aligned}
$$

$r \in \mathbb{N}_{0}$. Here $P_{k, 2 r+2}(t), k=1,2$ are defined as in (2.5), that is,

$$
\begin{aligned}
& P_{k, 2 r+2}(t)=-H_{k}(t)^{r+1}+\sum_{j=0}^{r}\left(2 a_{k}(t) g_{k, j}(t) S^{+}-h_{k, j}(t)\right) H_{k}(t)^{j}+g_{k, r+1}, \\
& \left.P_{k, 2 r+2}(t)\right|_{\operatorname{Ker}\left(\tau_{k}(t)-z\right)}=2 a_{k}(t) G_{k, r}(z, t) S^{+}-H_{k, r+1}(z, t)
\end{aligned}
$$

$\left\{g_{k, j}(n, t)\right\}_{0 \leq j \leq r},\left\{h_{k, j}(n, t)\right\}_{0 \leq j \leq r+1}$ are defined as in 2.4), and the polynomials $G_{k, r}(z, n, t), H_{k, r+1}(z, n, t)$ are defined as in (2.7). Moreover, we choose the same integration constants in $P_{1,2 r+2}(t)$ and $P_{2,2 r+2}(t)$ (i.e., $c_{1, \ell}=c_{2, \ell} \equiv c_{\ell}, 1 \leq \ell \leq r$ ).

Analogous to equation (2.8) one obtains that

$$
\frac{d}{d t} D(t)-\left[Q_{2 r+2}(t), D(t)\right]=0
$$

is equivalent to

$$
\begin{aligned}
\underline{K M}_{r}(\rho) & =\left(\mathrm{KM}_{r}(\rho)_{e}, \mathrm{KM}_{r}(\rho)_{o}\right) \\
& =\left(\begin{array}{c}
\dot{\rho}_{e}-\rho_{e}\left(g_{2, r+1}-g_{1, r+1}\right) \\
\dot{\rho}_{o}+\rho_{o}\left(g_{2, r+1}-g_{1, r+1}^{+}\right)
\end{array}\right)=0 .
\end{aligned}
$$

As in the Toda context (2.11), varying $r \in \mathbb{N}_{0}$ yields the Kac-van Moerbeke hierarchy (KM hierarchy) which we denote by

$$
\mathrm{KM}_{r}(\rho)=0, \quad r \in \mathbb{N}_{0} .
$$

Again the Lax equation (3.11) implies

Theorem 3.2. Let $\rho$ satisfy (H 3.1) and $\operatorname{KM}(\rho)=0$. Then the Lax equation 3.11) implies the existence of a unitary propagator $V_{r}(t, s)$ such that we have

$$
D(t)=V_{r}(t, s) D(s) V_{r}(t, s)^{-1}, \quad(t, s) \in \mathbb{R}^{2} .
$$

Thus all operators $D(t), t \in \mathbb{R}$ are unitarily equivalent.

And as in Theorem 2.4 we infer

Theorem 3.3. Suppose $\rho_{0} \in \ell^{\infty}(\mathbb{Z})$. Then there exists a unique integral curve $t \mapsto \rho(t)$ in $C^{\infty}\left(\mathbb{R}, \ell^{\infty}(\mathbb{Z})\right)$ of the Kac-van Moerbeke equations, that is, $\operatorname{KM}_{r}(\rho)=0$, such that $\rho(0)=\rho_{0}$.

As a simple consequence of Theorem 3.2 we have

$$
\frac{d}{d t} D(t)^{2}-\left[Q_{2 r+2}(t), D(t)^{2}\right]=0
$$


and observing

$$
D(t)^{2}=\left(\begin{array}{cc}
H_{1}(t) & 0 \\
0 & H_{2}(t)
\end{array}\right)
$$

yields the implication

$$
\mathrm{KM}_{r}(\rho)=0 \Rightarrow \mathrm{TL}_{r}\left(a_{k}, b_{k}\right)=0, \quad k=1,2,
$$

that is, given a solution $\rho$ of the $\mathrm{KM}_{r}$ equation (3.13), one obtains two solutions, $\left(a_{1}, b_{1}\right)$ and $\left(a_{2}, b_{2}\right)$, of the $\mathrm{TL}_{r}$ equations (2.11) related to each other by the Miuratype ([1]) transformations (3.6), 3.7). Note that due to $(\mathrm{H}, 3.1),\left(a_{1}, b_{1}\right)$ and $\left(a_{2}, b_{2}\right)$ both fulfill $(\mathrm{H}, 2.1)$.

In addition, we can define

$$
\phi_{1}(n, t)=-\frac{\rho_{e}(n, t)}{\rho_{o}(n, t)}, \quad \phi_{2}(n, t)=-\frac{\rho_{o}(n, t)}{\rho_{e}(n+1, t)} .
$$

This implies

$$
a_{k}(n, t) \phi_{k}(n, t)+\frac{a_{k}(n-1, t)}{\phi_{k}(n-1, t)}+b_{k}(n, t)=0,
$$

and

$$
\begin{aligned}
\frac{d}{d t} & \ln \phi_{k}(n, t)=-2 a_{k}(n, t)\left(g_{k, r}(n, t) \phi_{k}(n, t)+g_{k, r}(n+1, t) \phi_{k}(n, t)^{-1}\right) \\
& -2 b_{k}(n+1, t) g_{k, r}(n+1, t)+\left(g_{k, r+1}(n+1, t)-g_{k, r+1}(n, t)\right) \\
& -\left(h_{k, r}(n+1, t)-h_{k, r}(n, t)\right) .
\end{aligned}
$$

Hence we infer

$$
H_{k}(t) u_{k}(n, t)=0, \quad \frac{d}{d t} u_{k}(n, t)=P_{k, 2 r+2}(t) u_{k}(n, t)
$$

(in the weak sense, i.e., $u_{k}$ is not necessarily square summable), where

$$
\begin{aligned}
u_{k}(n, t)= & \exp \left(\int _ { t _ { 0 } } ^ { t } \left(2 a_{k}\left(n_{0}, x\right) g_{k, r}\left(n_{0}, x\right) \phi_{k}\left(n_{0}, x\right)-h_{k, r}\left(n_{0}, x\right)\right.\right. \\
& \left.\left.+g_{k, r+1}\left(n_{0}, x\right)\right) d x\right)\left\{\begin{array}{cc}
\prod_{m=n_{0}}^{n-1} \phi_{k}(m, t) & \text { for } n>n_{0} \\
1 & \text { for } n=n_{0} \\
\prod_{m=n}^{n_{0}-1} \phi_{k}(m, t)^{-1} & \text { for } n<n_{0}
\end{array} .\right.
\end{aligned}
$$

Furthermore, explicitly writing out (3.11) shows that if

$$
H_{k}(t) u_{k}(z, n, t)=z u_{k}(z, n, t), \quad \frac{d}{d t} u_{k}(z, n, t)=P_{k, 2 r+2}(t) u_{k}(z, n, t)
$$

holds (weakly) for $u_{1}(z, n, t)$ (resp. $\left.u_{2}(z, n, t)\right)$ then it also holds for $u_{2}(z, n, t)=$ $A u_{1}(z, n, t)$ (resp. $\left.u_{1}(z, n, t)=A^{*} u_{2}(z, n, t)\right)$.

Summarizing:

Theorem 3.4. Suppose $\rho$ satisfies (H.3.1) and $\operatorname{KM}_{r}(\rho)=0$. Then $\left(a_{k}, b_{k}\right), k=1,2$ satisfies (H 2.1) and $\mathrm{TL}_{r}\left(a_{k}, b_{k}\right)=0, k=1,2$. In addition, if $u_{1}(z, n, t)$ (resp. $u_{2}(z, n, t)$ ) is a weak solution of (3.23) then so is $u_{2}(z, n, t)=A u_{1}(z, n, t)$ (resp. $\left.u_{1}(z, n, t)=A^{*} u_{2}(z, n, t)\right)$. 


\section{Time evolution of solutions of the Jacobi equation}

The objective of this section is to investigate weak solutions of the system 2.15). As a first step we try to calculate the time evolution of the fundamental matrix $\Phi(z, n, t)$ corresponding to the difference equation $\tau(t) u=z u$, that is,

$$
\Phi(z, n, t)=\left(\begin{array}{cc}
c(z, n, t) & s(z, n, t) \\
c(z, n+1, t) & s(z, n+1, t)
\end{array}\right)
$$

is the matrix valued solution of $\tau(t) \Phi=z \Phi$ corresponding to the initial condition $\Phi(z, 0, t)=\mathbb{1}$. We assume that $a(t), b(t)$ satisfy $\mathrm{TL}_{r}(a, b)=0$. First observe, that (2.8) implies

$$
(H(t)-z)\left(\frac{d}{d t}-P_{2 r+2}(t)\right) \Phi(z, ., t)=0 .
$$

But this means

$$
\left(\frac{d}{d t}-P_{2 r+2}(t)\right) \Phi(z, ., t)=\Phi(z, ., t) C_{r}(z, t),
$$

for a certain matrix $C_{r}(z, t)$. If we evaluate the above expression at $n=0$ using $\Phi(z, 0, t)=\mathbb{1}$ we obtain

$$
\begin{aligned}
& C_{r}(z, t)=P_{2 r+2}(t) \Phi(z, 0, t) \\
& \quad=\left(\begin{array}{cc}
-H_{r+1}(z, 0, t) & 2 a(0, t) G_{r}(z, 0, t) \\
-2 a(0, t) G_{r}(z, 1, t) & 2(z-b(1, t)) G_{r}(z, 1, t)-H_{r+1}(z, 1, t)
\end{array}\right) .
\end{aligned}
$$

and hence

$$
\dot{\Phi}(z, n, t)=P_{2 r+2}(t) \Phi(z, n, t)+\Phi(z, n, t) C_{r}(z, t) .
$$

This result enables us to prove

Lemma 4.1. Assume (H,2.1) and suppose $\mathrm{TL}_{r}(a, b)=0$. Let $u_{0}(z, n)$ be a weak solution of $H(0) u_{0}=z u_{0}$. Then the system

$$
H(t) u(z, n, t)=z u(z, n, t), \quad \frac{d}{d t} u(z, n, t)=P_{2 r+2}(t) u(z, n, t),
$$

has a unique weak solution fulfilling the initial condition

$$
u(z, n, 0)=u_{0}(z, n) .
$$

If $u_{0}(z, n)$ is continuous (resp. holomorphic) with respect to $z$ then so is $u(z, n, t)$.

Furthermore, if $u_{1,2}(z, n, t)$ both solve (4.6) then

$W_{n}\left(u_{1}(z, t), u_{2}(z, t)\right)=a(n, t)\left(u_{1}(z, n, t) u_{2}(z, n+1, t)-u_{1}(z, n+1, t) u_{2}(z, n, t)\right)$, depends neither on $n$ nor on $t$.

Proof. Clearly, any solution $u(z, n, t)$ of the system (4.6) can be written as

$$
u(z, n, t)=u(z, 0, t) c(z, n, t)+u(z, 1, t) s(z, n, t),
$$

and from 4.5 we infer that 4.6 is equivalent to

$$
\left(\begin{array}{c}
\dot{u}(z, 0, t) \\
\dot{u}(z, 1, t)
\end{array}\right)=-C_{r}(z, t)\left(\begin{array}{l}
u(z, 0, t) \\
u(z, 1, t)
\end{array}\right), \quad\left(\begin{array}{l}
u(z, 0,0) \\
u(z, 1,0)
\end{array}\right)=\left(\begin{array}{l}
u_{0}(z, 0) \\
u_{0}(z, 1)
\end{array}\right),
$$

which proves the first assertion. The second is a straightforward calculation using 2.6 and $\dot{a}=a\left(H_{r+1}^{+}+H_{r+1}-2\left(z-b^{+}\right) G_{r}^{+}\right)$. 
In the special case $r=0$ this result was first given in [8, Lemma 2.4. Next, let us verify some additional properties of solutions of (4.6). The following result was first observed in [6] for the special case $r=0, \lambda<\sigma(H)$.

Lemma 4.2. Let $u_{ \pm, 0}(z, n)$ be a solution of $H(0) u=z u, z \in \mathbb{C}$ which is square summable near $\pm \infty$. Then the solution $u_{ \pm}(z, n, t)$ of the system (4.6) with initial data $u_{ \pm, 0}(z, n)$ is square summable near $\pm \infty$ for all $t$.

Proof. We only prove the - case (the + case follows from reflection) and drop $z$ for notational simplicity. By Lemma 4.1 we have a solution $u(n, t)$ of 4.6 with initial condition $u(n, 0)=u_{+, 0}(n)$ and hence

$$
S(n, t)=S(n, 0)+2 \int_{0}^{t} \operatorname{Re} \sum_{j=-n}^{0} \overline{u(j, s)} P_{2 r+2}(s) u(j, s) d s,
$$

where $S(n, t)=\sum_{j=-n}^{0}|u(j, t)|^{2}$. Next, by boundedness of $a(t), b(t)$ we can find a constant $C>0$ such that $4\left|H_{r+1}(n, t)\right| \leq C$ and $8\left|a(n, t) G_{r}(n, t)\right| \leq C$. Using 2.6) and Cauchy's inequality implies

$$
\left|\sum_{j=-n}^{0} \overline{u(j, s)} P_{2 r+2}(s) u(j, s)\right| \leq \frac{C}{2}\left(|u(1, s)|^{2}+S(n, s)\right) .
$$

Invoking Gronwall's inequality shows

$$
S(n, t) \leq\left(S(n, 0)+C \int_{0}^{t}|u(1, s)|^{2} \mathrm{e}^{-C s} d s\right) \mathrm{e}^{C t}
$$

and letting $n \rightarrow \infty$ completes the proof.

We finish this section by investigating positive solutions of 4.6p.

Lemma 4.3. Suppose $\lambda \leq \sigma(H(0))$ and $a(n, t)<0$. Then $u_{0}(\lambda, n)>0$ implies that the solution $u(\lambda, n, t)$ of (4.6) with initial condition $u_{0}(\lambda, n)$ is positive.

Proof. Shifting $H(t) \rightarrow H(t)-\lambda$ we can assume $\lambda=0$. Now use $u_{0}(0, n)>$ 0 to define $\rho_{0}(n)$ by $\rho_{0, o}(n)=-\sqrt{-a(n, 0) u_{0}(0, n) / u_{0}(0, n+1)}$ and $\rho_{0, e}(n)=$ $\sqrt{-a(n, 0) u_{0}(0, n+1) / u_{0}(0, n)}$. By Theorem 3.3 we have a corresponding solution $\rho(n, t)$ of the KM hierarchy and hence (by (3.17)) two solutions $a_{j}(n, t), b_{j}(n, t)$ of the TL hierarchy. Since $a_{1}(n, 0)=a(n, 0)$ and $b_{1}(n, 0)=b(n, 0)$ we infer $a_{1}(n, t)=a(n, t)$ and $b_{1}(n, t)=b(n, t)$ by uniqueness (Theorem 2.4). Finally, we conclude $u(0, n, t)=u_{0}\left(0, n_{0}\right) u_{1}(n, t)>0$ (with $u_{1}(n, t)$ as in 3.22$)$ again by uniqueness (Theorem 4.1).

In the special case $r=0$ and under the additional assumption $b(n, 0) / a(n, 0)=$ $o(|n|)$ as $n \rightarrow \pm \infty$, this result has first been proven in 8, Lemma 2.6. We remark that for $\lambda<\sigma(H(0))$ it also follows from Lemma 4.2 and [17, Lemma A.2.

Recall that positive solutions of $\tau(t) u=\lambda u(a(n, t)<0)$ can be characterized in terms of minimal (also principal or recessive) positive solutions ([2, 7], 12]). Suppose $H(t)-\lambda \geq 0$, then the minimal positive solution $u_{+}(\lambda, n, t)$ near $+\infty$ is determined by

$$
\frac{u_{+}(\lambda, n, t)}{u(\lambda, n, t)}<\frac{u_{+}(\lambda, 0, t)}{u(\lambda, 0, t)}, n \in \mathbb{N}, \quad \lim _{n \rightarrow+\infty} \frac{u_{+}(\lambda, n, t)}{u(\lambda, n, t)}=0
$$


for any linearly independent solution $u(\lambda, n, t)$ with $u(\lambda, n, t)>0, n \in \mathbb{N}$. Similarly for $u_{-}(\lambda, n, t)$, the minimal solution near $-\infty$.

Two cases may occur

(i). $u_{-}(\lambda, n, t), u_{+}(\lambda, n, t)$ are linearly dependent and there is only one (up to constant multiples) positive solution. $(H(t)-\lambda$ is critical.)

(ii). $u_{-}(\lambda, n, t), u_{+}(\lambda, n, t)$ are linearly dependent and

$$
u_{\sigma}(\lambda, n, t)=\frac{1+\sigma}{2} u_{+}(\lambda, n, t)+\frac{1-\sigma}{2} u_{-}(\lambda, n, t),
$$

is positive if and only if $\sigma \in[-1,1]$. ( $H(t)-\lambda$ is subcritical.)

In case (ii) one can easily show that for two positive solutions $u_{j}(\lambda, n, t), j=1,2$ we have

$$
u_{\sigma}(\lambda, n, t)=\frac{1+\sigma}{2} u_{1}(\lambda, n, t)+\frac{1-\sigma}{2} u_{2}(\lambda, n, t)>0 \quad \Leftrightarrow \quad \sigma \in[-1,1],
$$

if and only if $u_{1,2}$ equal $u_{ \pm}$up to constant (w.r.t. $n$ ) multiples.

Lemma 4.4. Let $\lambda \leq \sigma(H(0))$ and $a(n, t)<0$. Suppose $u(\lambda, n, t)$ solves $\sqrt{4.6})$ and is a minimal positive solution for one $t=t_{0}$, then this holds for all $t \in \mathbb{R}$. In particular, $H(t)-\lambda$ is critical (resp. subcritical) for all $t \in \mathbb{R}$ if and only if it is critical for one $t=t_{0}$.

Proof. Since linear independence and positivity is preserved by the system 4.6 (by (4.8) and Lemma 4.3 $H(t)-\lambda$ is critical (resp. subcritical) for all $t \in \mathbb{R}$ if and only if it is critical for one $t=t_{0}$. If $H(t)-\lambda$ is subcritical we note that the characterization (4.16) of minimal solutions is independent of $t$. Hence it could only happen that $u_{+}(\lambda, n, t)$ and $u_{-}(\lambda, n, t)$ change place during time evolution. But this would imply $u_{+}(\lambda, n, t)$ and $u_{-}(\lambda, n, t)$ are linearly dependent at some intermediate time contradicting $H(t)-\lambda$ subcritical.

That minimal solutions remain minimal is suggested by the notation chosen in [8], Theorem 2.9 (however, no proof is given). The remaining assertion for the special case $r=0$ with the additional assumption $b(n, 0) / a(n, 0)=o(|n|)$ as $n \rightarrow \pm \infty$ corresponds to [8, Lemma 2.10. Again, for $\lambda<\sigma(H)$ the lemma already follows from Lemma 4.2 .

In particular, this shows that the choice in (5.7) below is exhaustive.

\section{5. $N$-SOLITON SOLUTIONS ON AN ARBITRARY BACKGROUND}

In Theorem 3.4 we saw, that from one solution $\rho$ of $\operatorname{KM}_{r}(\rho)=0$ we can get two solutions $\left(a_{1}, b_{1}\right),\left(a_{2}, b_{2}\right)$ of $\mathrm{TL}_{r}(a, b)=0$. In this section we want to invert this process.

Suppose $(a, b)$ satisfies $(\mathrm{H} 2.1), a(n, t)<0$ and $\mathrm{TL}_{r}(a, b)=0$. Suppose $\lambda_{1} \leq$ $\sigma(H(0))$ and let $u_{ \pm}\left(\lambda_{1}, n, t\right)>0$ be the minimal positive solutions of 4.6 found in Lemma 4.4 and set

$$
u_{\sigma_{1}}\left(\lambda_{1}, n, t\right)=\frac{1+\sigma_{1}}{2} u_{+}\left(\lambda_{1}, n, t\right)+\frac{1-\sigma_{1}}{2} u_{-}\left(\lambda_{1}, n, t\right) .
$$


Note that the dependence on $\sigma_{1}$ will drop out in what follows if $u_{+}\left(\lambda_{1}, n, t\right)$ and $u_{-}\left(\lambda_{1}, n, t\right)$ are linearly dependent (for one and hence for all $t$ ). Now define

$$
\rho_{\sigma_{1}, o}(n, t)=-\sqrt{-\frac{a(n, t)}{\phi_{\sigma_{1}}\left(\lambda_{1}, n, t\right)}}, \quad \rho_{\sigma_{1}, e}(n, t)=\sqrt{-a(n, t) \phi_{\sigma_{1}}\left(\lambda_{1}, n, t\right)},
$$

where $\phi_{\sigma_{1}}\left(\lambda_{1}, n, t\right)=u_{\sigma_{1}}\left(\lambda_{1}, n+1, t\right) / u_{\sigma_{1}}\left(\lambda_{1}, n, t\right)$.

Then, proceeding as in the proof of Lemma 4.3 shows that the sequence

$$
\rho_{\sigma_{1}}(n, t)=\left\{\begin{array}{l}
\rho_{\sigma_{1}, e}(m, t) \text { for } n=2 m \\
\rho_{\sigma_{1}, o}(m, t) \text { for } n=2 m+1
\end{array},\right.
$$

fulfills $\left(\mathrm{H} 3.1\right.$ and $\mathrm{KM}_{r}(\rho)=0$. Hence by (3.17)

$$
a_{\sigma_{1}}(n, t)=\rho_{\sigma_{1}, e}(n+1, t) \rho_{\sigma_{1}, o}(n, t), \quad b_{\sigma_{1}}(n, t)=\rho_{\sigma_{1}, e}(n, t)^{2}+\rho_{\sigma_{1}, o}(n, t)^{2}
$$

satisfy $\mathrm{TL}_{r}\left(a_{\sigma_{1}}, b_{\sigma_{1}}\right)=0$.

We summarize this result in our first main theorem.

Theorem 5.1. Suppose $(a, b)$ satisfies (H,2.1) and $\mathrm{TL}_{r}(a, b)=0$. Pick $\lambda_{1} \leq$ $\sigma(H(0)), \sigma_{1} \in[-1,1]$ and let $u_{ \pm}\left(\lambda_{1}, n, t\right)$ be the minimal positive solutions of (4.6). Then the sequences

$$
\begin{aligned}
& a_{\sigma_{1}}(n, t)=-\sqrt{\frac{a(n, t) a(n+1, t) u_{\sigma_{1}}\left(\lambda_{1}, n, t\right) u_{\sigma_{1}}\left(\lambda_{1}, n+2, t\right)}{u_{\sigma_{1}}\left(\lambda_{1}, n+1, t\right)^{2}}}, \\
& b_{\sigma_{1}}(n, t)=b(n, t)-\partial^{*} \frac{a(n, t) u_{\sigma_{1}}\left(\lambda_{1}, n, t\right)}{u_{\sigma_{1}}\left(\lambda_{1}, n+1, t\right)}
\end{aligned}
$$

with

$$
u_{\sigma_{1}}\left(\lambda_{1}, n, t\right)=\frac{1+\sigma_{1}}{2} u_{+}\left(\lambda_{1}, n, t\right)+\frac{1-\sigma_{1}}{2} u_{-}\left(\lambda_{1}, n, t\right),
$$

satisfy (H.2.1) and $\mathrm{TL}_{r}\left(a_{\sigma_{1}}, b_{\sigma_{1}}\right)=0$. Here $\partial^{*} f(n)=f(n-1)-f(n)$. In addition,

$$
\frac{a(n, t)\left(u_{\sigma_{1}}\left(\lambda_{1}, n, t\right) u(z, n+1, t)-u_{\sigma_{1}}\left(\lambda_{1}, n+1, t\right) u(z, n, t)\right)}{\sqrt{-a(n, t) u_{\sigma_{1}}\left(\lambda_{1}, n, t\right) u_{\sigma_{1}}\left(\lambda_{1}, n+1, t\right)}}
$$

satisfies $H_{\sigma_{1}} u=z u$ and $d / d t u=P_{\sigma_{1}, 2 r+2} u$ (weakly) (in obvious notation) and $\rho_{\sigma_{1}}(n, t)$ defined as in (5.3) satisfies (H.3.1) and $\mathrm{KM}_{r}(\rho)=0$.

The special case $r=0$ was first proven in $[8$, Theorem 2.9. The general case is stated in [3], Theorem 7.2 without proof.

Remark 5.2. (i). Alternatively, one could give a direct algebraic proof of the above theorem using $H_{\sigma_{1}}^{j+1}=A_{\sigma_{1}} H^{j} A_{\sigma_{1}}^{*}$ to express the quantities $g_{\sigma_{1}, j}, h_{\sigma_{1}, j}$ in terms of $g_{j}, h_{j}$.

(ii). We have omitted the requirement $a(n, t)<0$ since the formulas for $a_{\sigma_{1}}, b_{\sigma_{1}}$ are actually independent of the sign of $a(n, t)$. In addition, we could even allow $\lambda_{1} \geq \sigma(H(0))$. However, $\rho_{\sigma_{1}, e}(n, t)$ and $\rho_{\sigma_{1}, o}(n, t)$ would be purely imaginary in this case.

Iterating this procedure (cf. [], Theorem 3.1) gives

Theorem 5.3. Let $a(t), b(t)$ satisfy (H 2.1) and $\mathrm{TL}_{r}(a, b)=0$. Let $H(t)$ be the corresponding Jacobi operators and choose $(N \in \mathbb{N})$

$$
\lambda_{N}<\cdots<\lambda_{2}<\lambda_{1} \leq \sigma(H(0)), \quad \sigma_{\ell} \in[-1,1], \quad 1 \leq \ell \leq N, N \in \mathbb{N} .
$$


Suppose $u_{ \pm}(\lambda, n, t)$, are the principal solutions of 4.6). Then the sequences

$$
\begin{aligned}
a_{\sigma_{1}, \ldots, \sigma_{N}}(n, t)= & -\sqrt{a(n, t) a(n+N, t)} \\
& \times \frac{\sqrt{C_{n}\left(U_{\sigma_{1}, \ldots, \sigma_{N}}(t)\right) C_{n+2}\left(U_{\sigma_{1}, \ldots, \sigma_{N}}(t)\right)}}{C_{n+1}\left(U_{\sigma_{1}, \ldots, \sigma_{N}}(t)\right)}, \\
b_{\sigma_{1}, \ldots, \sigma_{N}}(n, t)= & b(n, t)+\partial^{*} a(n, t) \frac{D_{n}\left(U_{\sigma_{1}, \ldots, \sigma_{N}}(t)\right)}{C_{n+1}\left(U_{\sigma_{1}, \ldots, \sigma_{N}}(t)\right)}
\end{aligned}
$$

satisfy $\mathrm{TL}_{r}\left(a_{\sigma_{1}, \ldots, \sigma_{N}}, b_{\sigma_{1}, \ldots, \sigma_{N}}\right)=0$. Here $C_{n}$ denotes the $n$-dimensional Casoratian

$$
\begin{aligned}
& C_{n}\left(u_{1}, \ldots, u_{N}\right)=\operatorname{det}\left(u_{i}(n+j-1)\right)_{1 \leq i, j \leq N}, \\
& D_{n}\left(u_{1}, \ldots, u_{N}\right)=\operatorname{det}\left(\begin{array}{cc}
u_{i}(n), & j=1 \\
u_{i}(n+j), & j>1
\end{array}\right)_{1 \leq i, j \leq N}
\end{aligned}
$$

and $\left(U_{\sigma_{1}, \ldots, \sigma_{N}}(t)\right)=\left(u_{\sigma_{1}}^{1}(t), \ldots, u_{\sigma_{N}}^{N}(t)\right)$ with

$$
u_{\sigma_{\ell}}^{\ell}(n, t)=\frac{1+\sigma_{\ell}}{2} u_{+}\left(\lambda_{\ell}, n, t\right)+(-1)^{\ell+1} \frac{1-\sigma_{\ell}}{2} u_{-}\left(\lambda_{\ell}, n, t\right) .
$$

Defining

$$
\begin{aligned}
& \rho_{\sigma_{1}, \ldots, \sigma_{N}, o}(n, t)= \\
& -\sqrt{-a(n, t) \frac{C_{n+2}\left(U_{\sigma_{1}, \ldots, \sigma_{N-1}}(t)\right) C_{n}\left(U_{\sigma_{1}, \ldots, \sigma_{N}}(t)\right)}{C_{n+1}\left(U_{\sigma_{1}, \ldots, \sigma_{N-1}}(t)\right) C_{n+1}\left(U_{\sigma_{1}, \ldots, \sigma_{N}}(t)\right)}}, \\
& \rho_{\sigma_{1}, \ldots, \sigma_{N}, e}(n, t)= \\
& \sqrt{-a(n+N-1, t) \frac{C_{n}\left(U_{\sigma_{1}, \ldots, \sigma_{N-1}}(t)\right) C_{n+1}\left(U_{\sigma_{1}, \ldots, \sigma_{N}}(t)\right)}{C_{n+1}\left(U_{\sigma_{1}, \ldots, \sigma_{N-1}}(t)\right) C_{n}\left(U_{\sigma_{1}, \ldots, \sigma_{N}}(t)\right)}},
\end{aligned}
$$

the corresponding sequence $\rho_{\sigma_{1}, \ldots, \sigma_{N}}(n)$ solves $\operatorname{KM}_{r}\left(\rho_{\sigma_{1}, \ldots, \sigma_{N}}\right)=0$.

Remark 5.4. The formula for $b_{\sigma_{1}, \ldots, \sigma_{N}}(n, t)$ is new. It can be obtained from the one given in [6] (equation (3.6)) by observing that this formula holds with $\lambda_{N}$ replaced by arbitrary $z \in \mathbb{C}$ and performing the limit $z \rightarrow \infty$.

Clearly, if we drop the requirement $\lambda \leq \sigma(H(0))$ the solution $u_{\sigma_{1}}\left(\lambda_{1}, n, t\right)$ used to perform the factorization will no longer be positive. Hence the sequences $a_{\sigma_{1}}(n, t)$, $b_{\sigma_{1}}(n, t)$ can be complex valued and singular. Nevertheless there are two situations where a second factorization step produces again real-valued non-singular solutions.

Firstly we perform two steps with $\lambda_{1,2}$ in the same spectral gap of $H(0)$ (see [16] for a detailed spectral analysis of this method).

Theorem 5.5. Suppose $(a, b)$ satisfies (H,2.1) and $\mathrm{TL}_{r}(a, b)=0$. Pick $\lambda_{1,2}, \sigma_{1,2} \in$ $\{ \pm 1\}$ and let $\lambda_{1,2}$ lie in the same spectral gap of $H(0)\left(\left(\lambda_{1}, \sigma_{1}\right) \neq\left(\lambda_{2},-\sigma_{2}\right)\right.$ to make sure we get something new). Then the sequences

$$
\begin{aligned}
& a_{\sigma_{1}, \sigma_{2}}(n, t)=a(n, t) \sqrt{\frac{W_{\sigma_{1}, \sigma_{2}}(n-1, t) W_{\sigma_{1}, \sigma_{2}}(n+1, t)}{W_{\sigma_{1}, \sigma_{2}}(n, t)^{2}}}, \\
& b_{\sigma_{1}, \sigma_{2}}(n, t)=b(n, t)-\partial^{*} \frac{a(n, t) u_{\sigma_{1}}\left(\lambda_{1}, n, t\right) u_{\sigma_{2}}\left(\lambda_{2}, n+1, t\right)}{W_{\sigma_{1}, \sigma_{2}}(n, t)}
\end{aligned}
$$


are real-valued non-singular solutions of $\mathrm{TL}_{r}\left(a_{\sigma_{1}, \sigma_{2}}, b_{\sigma_{1}, \sigma_{2}}\right)=0$. Here

$$
W_{\sigma_{1}, \sigma_{2}}(n, t)=\left\{\begin{array}{ll}
\frac{W_{n}\left(u_{\sigma_{1}}\left(\lambda_{1}, t\right), u_{\sigma_{2}}\left(\lambda_{2}, t\right)\right)}{\lambda_{2}-\lambda_{1}}, & \lambda_{1} \neq \lambda_{2} \\
\sum_{m=\sigma_{1} \infty}^{n} u_{\sigma_{1}}\left(\lambda_{1}, m, t\right)^{2}, & \left(\lambda_{1}, \sigma_{1}\right)=\left(\lambda_{2}, \sigma_{2}\right)
\end{array},\right.
$$

where $\sum_{m=+\infty}^{n}=-\sum_{m=n+1}^{\infty}$.

In addition, the sequence

$$
\frac{W_{\sigma_{1}, \sigma_{2}}(n, t) u(z, n, t)-\frac{1}{z-\lambda_{1}} u_{\sigma_{2}}\left(\lambda_{2}, n, t\right) W_{n}\left(u_{\sigma_{1}}\left(\lambda_{1}, t\right), u(z, t)\right)}{\sqrt{W_{\sigma_{1}, \sigma_{2}}(n-1, t) W_{\sigma_{1}, \sigma_{2}}(n, t)}},
$$

satisfies $H_{\sigma_{1}, \sigma_{2}}(t) u=z u, d / d t u=P_{\sigma_{1}, \sigma_{2}, 2 r+2}(t) u$ (weakly).

Proof. Theorem 4.6 of [17] implies $W_{\sigma_{1}, \sigma_{2}}(n, t) W_{\sigma_{1}, \sigma_{2}}(n+1, t)>0$ and hence the sequences $a_{\sigma_{1}, \sigma_{2}}(t), b_{\sigma_{1}, \sigma_{2}}(t)$ satisfy $(\mathrm{H}[2.1)$ (see also [16] $)$. The rest follows from the previous theorem (with $N=2$ ) as follows. Replace $\lambda_{1}$ by $z \in\left(\lambda_{1}-\varepsilon, \lambda_{1}+\varepsilon\right.$ ) and observe that $a_{\sigma_{1}, \sigma_{2}}(n, t), b_{\sigma_{1}, \sigma_{2}}(n, t)$ and $\dot{a}_{\sigma_{1}, \sigma_{2}}(n, t), \dot{b}_{\sigma_{1}, \sigma_{2}}(n, t)$ are meromorphic with respect to $z$. From the algebraic structure we have simply performed two single commutation steps. Hence, provided Theorem 5.1 applies to this more general setting of meromorphic solutions, we can conclude that our claims hold except for a discrete set with respect to $z$ where the intermediate operators are ill-defined due to singularities of the coefficients. However, the proof of Theorem 5.1 uses these intermediate operators and in order to see that Theorem 5.1 still holds, one has to resort to the direct algebraic proof outlined in Remark 5.2(i). Continuity with respect to $z$ takes care of the remaining points.

To the best of our knowledge Theorem 5.5 is novel even in the case of the first Toda equation $r=0$. Secondly, we consider again two commutation steps but now with $\lambda_{1}=\lambda_{2}$.

Theorem 5.6. Suppose $(a, b)$ satisfies (H, 2.1) and $\mathrm{TL}_{r}(a, b)=0$. Pick $\lambda_{1}$ in a spectral gap of $H(0)$ and $\gamma_{1} \in\left[-\left\|u_{-}\left(\lambda_{1}\right)\right\|^{-2}, \infty\right) \cup\{\infty\}$. Then the sequences

$$
\begin{aligned}
& a_{\gamma_{1}}(n, t)=a(n, t) \frac{\sqrt{c_{\gamma_{1}}\left(\lambda_{1}, n-1, t\right) c_{\gamma_{1}}\left(\lambda_{1}, n+1, t\right)}}{c_{\gamma_{1}}\left(\lambda_{1}, n, t\right)}, \\
& b_{\gamma_{1}}(n, t)=b(n, t)-\partial^{*} \frac{a(n, t) u_{-}\left(\lambda_{1}, n, t\right) u_{-}\left(\lambda_{1}, n+1, t\right)}{c_{\gamma_{1}}\left(\lambda_{1}, n, t\right)} .
\end{aligned}
$$

satisfy $\operatorname{TL}\left(a_{\gamma_{1}}, b_{\gamma_{1}}\right)=0$, where

$$
c_{\gamma_{1}}\left(\lambda_{1}, n, t\right)=\frac{1}{\gamma_{1}}+\sum_{m=-\infty}^{n} u_{-}\left(\lambda_{1}, m, t\right)^{2} .
$$

In addition, the sequence

$$
\frac{c_{\gamma_{1}}\left(\lambda_{1}, n, t\right) u(z, n, t)-\frac{1}{z-\lambda_{1}} u_{-}\left(\lambda_{1}, n, t\right) W_{n}\left(u_{-}\left(\lambda_{1}, t\right), u(z, t)\right)}{\sqrt{c_{\gamma_{1}}\left(\lambda_{1}, n-1, t\right) c_{\gamma_{1}}\left(\lambda_{1}, n, t\right)}},
$$

satisfies $H_{\gamma_{1}}(t) u=z u, d / d t u=P_{\gamma_{1}, 2 r+2}(t) u$ (weakly).

Proof. Following [6], p256 we can obtain the double commutation method from two single commutation steps. We pick $\sigma_{1}=-1$ for the first factorization. Considering 
$A_{\sigma_{1}} u_{-}(z, n+1, t) /\left(z-\lambda_{1}\right)$ and performing the limit $z \rightarrow \lambda_{1}$ shows that

$$
v\left(\lambda_{1}, n, t\right)=\frac{c_{\gamma_{1}}\left(\lambda_{1}, n, t\right)}{\sqrt{-a(n, t) u_{-}\left(\lambda_{1}, n, t\right) u_{-}\left(\lambda_{1}, n+1, t\right)}}
$$

is a solution of the new (singular) operator which can be used to perform a second factorization. The resulting operator is associated with $a_{\gamma_{1}}, b_{\gamma_{1}}$. Now argue as before.

As already mentioned before, the special case $r=0$ was first given in [6]. Again we point out that one can also prove this theorem directly as follows. Without restriction we choose $\lambda_{1}=0$. Then one computes

$$
\begin{aligned}
& \frac{d}{d t} c_{\gamma_{1}}(0, n, t)=2 a(n, t)^{2}\left(g_{r-1}(n+1, t) u_{-}(0, n, t)^{2}+g_{r-1}(n, t) u_{-}(0, n+1, t)^{2}\right) \\
& (5.26) \quad+2 h_{r-1}(n, t) a(n, t) u_{-}(0, n, t) u_{-}(0, n+1, t)
\end{aligned}
$$

and it remains to relate $g_{\gamma_{1}, j}, h_{\gamma_{1}, j}$ and $g_{j}, h_{j}$. Since these quantities arise as coefficients of the Neumann expansion of the respective Green functions it suffices to relate the Green functions of $H_{\gamma_{1}}$ and $H$. This can be done using [6], Lemma 4.6 (compare [6], (2.40)).

Iterating this procedure (cf. [6], Theorem 6.1) gives

Theorem 5.7. Let $a(n, t), b(n, t)$ satisfy (H,2.1) and $\mathrm{TL}_{r}(a, b)=0$. and let $H(t)$ be the corresponding Jacobi operators. Let $\lambda_{j} \in \rho(H(0)), \gamma_{j} \in\left[-\left\|u_{-}\left(\lambda_{j}\right)\right\|^{-2}, \infty\right) \cup$ $\{\infty\}, 1 \leq j \leq N$ and

$$
\frac{d}{d t} u_{-}\left(\lambda_{j}, n, t\right)=P_{2 r+2}(t) u_{-}\left(\lambda_{j}, n, t\right) .
$$

We define the following matrices $(1 \leq \ell \leq N)$

$$
\begin{gathered}
C^{N}(n, t)=\left(\frac{\delta_{i j}}{\gamma_{i}}+\sum_{m=-\infty}^{n} u_{-}\left(\lambda_{i}, m, t\right) u_{-}\left(\lambda_{j}, m, t\right)\right)_{1 \leq i, j \leq N}, \\
D^{N}(n, t)=\left(\begin{array}{cl}
C^{N}(n, t)_{i, j}, & i, j \leq N \\
u_{-}\left(\lambda_{j}, n-1, t\right), & j \leq N, i=N+1 \\
u_{-}\left(\lambda_{i}, n, t\right), & i \leq N, j=N+1 \\
0, & i=j=N+1
\end{array}\right)_{1 \leq i, j \leq N+1} .
\end{gathered}
$$

Then the sequences

$$
\begin{aligned}
& a_{\gamma_{1}, \ldots, \gamma_{N}}(n, t)=a(n, t) \frac{\sqrt{\operatorname{det} C^{N}(n-1, t) \operatorname{det} C^{N}(n+1, t)}}{\operatorname{det} C^{N}(n, t)}, \\
& b_{\gamma_{1}, \ldots, \gamma_{N}}(n, t)=b(n, t)-\partial^{*} a(n, t) \frac{\operatorname{det} D^{N}(n+1, t)}{\operatorname{det} C^{N}(n, t)}
\end{aligned}
$$

satisfy $\mathrm{TL}_{r}\left(a_{\gamma_{1}, \ldots, \gamma_{N}}, b_{\gamma_{1}, \ldots, \gamma_{N}}\right)=0$.

Remark 5.8. (i). The formulas of [6] have been slightly rephrased to include the limit $\gamma_{j}=\infty$. In addition, the formula for $b_{\gamma_{1}, \ldots, \gamma_{N}}(n, t)$ is new. It can be obtained from the one given in [6] (equation (6.9)) by observing that this formula holds with $\lambda_{N}$ replaced by arbitrary $z \in \mathbb{C}$ and performing the limit $z \rightarrow \infty$.

(ii). The limit $N \rightarrow \infty$ can be performed as in [14. 
We conclude this section with an example; the $N$-soliton solutions of the TL and KM hierarchies.

We take the constant solution of the Toda hierarchy

$$
a_{0}(n, t)=\frac{1}{2}, \quad b_{0}(n, t)=0,
$$

as our background. If $H_{0}, P_{0,2 r+2}$ denotes the associated Lax pair we obtain (5.33)

$$
H_{0}(t) u_{0, \pm}(z, n, t)=z u_{0, \pm}(z, n, t), \quad \frac{d}{d t} u_{0, \pm}(z, n, t)=P_{0,2 r+2}(t) u_{0, \pm}(z, n, t),
$$

where

$$
u_{0, \pm}(z, n, t)=k^{ \pm n} \exp \left( \pm \frac{\alpha_{r}(k) t}{2}\right), \quad k=z-\sqrt{z^{2}-1},|k| \leq 1
$$

and

$$
\alpha_{r}(k)=2\left(k G_{0, r}(z)-H_{r+1,0}(z)\right)=\left(k-k^{-1}\right) G_{0, r}(z) .
$$

Explicitly we have

$$
\begin{aligned}
\alpha_{0}(k)= & k-k^{-1}, \\
\alpha_{1}(k)= & \frac{k^{2}-k^{-2}}{2}+c_{1}\left(k-k^{-1}\right), \\
& \text { etc. }
\end{aligned}
$$

Then the $N$-soliton solution of the Toda hierarchy is given by

$$
\begin{aligned}
a_{0, \sigma_{1}, \ldots, \sigma_{N}}(n, t) & =\frac{\sqrt{C_{n}\left(U_{\sigma_{1}, \ldots, \sigma_{N}}(t)\right) C_{n+2}\left(U_{\sigma_{1}, \ldots, \sigma_{N}}(t)\right)}}{2 C_{n+1}\left(U_{\sigma_{1}, \ldots, \sigma_{N}}(t)\right)}, \\
b_{0, \sigma_{1}, \ldots, \sigma_{N}}(n, t) & =\partial^{*} \frac{D_{n}\left(U_{\sigma_{1}, \ldots, \sigma_{N}}(t)\right)}{2 C_{n+1}\left(U_{\sigma_{1}, \ldots, \sigma_{N}}(t)\right)}
\end{aligned}
$$

where $\left(U_{0, \sigma_{1}, \ldots, \sigma_{N}}(t)\right)=\left(u_{\sigma_{1}}^{1}(t), \ldots, u_{\sigma_{N}}^{N}(t)\right)$ with

$$
u_{0, \sigma_{j}}^{j}(n, t)=k_{j}^{n}+(-1)^{\ell+1} \frac{1-\sigma_{j}}{1+\sigma_{j}} \exp \left(\alpha_{r}\left(k_{j}\right) t\right) k_{\ell}^{-n}, \quad k_{j}=\lambda_{j}-\sqrt{\lambda_{j}^{2}-1} .
$$

The corresponding $N$-soliton solution $\rho_{\sigma_{1}, \ldots, \sigma_{N}}(n)$ of the Kac-van Moerbeke hierarchy reads

$$
\begin{aligned}
& \rho_{0, \sigma_{1}, \ldots, \sigma_{N}, o}(n, t)= \\
& -\sqrt{-\frac{C_{n+2}\left(U_{0, \sigma_{1}, \ldots, \sigma_{N-1}}(t)\right) C_{n}\left(U_{0, \sigma_{1}, \ldots, \sigma_{N}}(t)\right)}{2 C_{n+1}\left(U_{0, \sigma_{1}, \ldots, \sigma_{N-1}}(t)\right) C_{n+1}\left(U_{0, \sigma_{1}, \ldots, \sigma_{N}}(t)\right)}}, \\
& \rho_{0, \sigma_{1}, \ldots, \sigma_{N}, e}(n, t)= \\
& \sqrt{-\frac{C_{n}\left(U_{0, \sigma_{1}, \ldots, \sigma_{N-1}}(t)\right) C_{n+1}\left(U_{0, \sigma_{1}, \ldots, \sigma_{N}}(t)\right)}{2 C_{n+1}\left(U_{0, \sigma_{1}, \ldots, \sigma_{N-1}}(t)\right) C_{n}\left(U_{0, \sigma_{1}, \ldots, \sigma_{N}}(t)\right)}} .
\end{aligned}
$$

Introducing the time dependent norming constants

$$
\gamma_{j}(t)=\gamma_{j} \exp \left(-\alpha_{r}\left(k_{j}\right) t\right)
$$


we obtain the following alternate expression for the $N$-soliton solution of the Toda hierarchy

$$
\begin{aligned}
& a_{0, \gamma_{1}, \ldots, \gamma_{N}}(n, t)=\frac{\sqrt{\operatorname{det} C_{0}^{N}(n-1, t) \operatorname{det} C_{0}^{N}(n+1, t)}}{2 \operatorname{det} C_{0}^{N}(n, t)}, \\
& b_{0, \gamma_{1}, \ldots, \gamma_{N}}(n, t)=-\partial^{*} \frac{\operatorname{det} D_{0}^{N}(n+1, t)}{2 \operatorname{det} C_{0}^{N}(n, t)},
\end{aligned}
$$

where

$$
\begin{gathered}
C_{0}^{N}(n, t)=\left(\frac{\delta_{i j}}{\gamma_{j}(t)}+\frac{\left(k_{i} k_{j}\right)^{-n}}{1-k_{i} k_{j}}\right)_{1 \leq i, j \leq N}, \\
D_{0}^{N}(n, t)=\left(\begin{array}{cl}
C_{0}^{N}(n, t)_{i, j}, & i, j \leq N \\
k_{j}^{1-n}, & j \leq N, i=N+1 \\
k_{i}^{-n}, & i \leq N, j=N+1 \\
0, & i=j=N+1
\end{array}\right)_{1 \leq i, j \leq N+1} .
\end{gathered}
$$

The sequences $a_{0, \gamma_{1}, \ldots, \gamma_{N}}, b_{0, \gamma_{1}, \ldots, \gamma_{N}}$ coincide with $a_{0, \sigma_{1}, \ldots, \sigma_{N}}, b_{0, \sigma_{1}, \ldots, \sigma_{N}}$ provided (cf. 8], Lemma 3.2 or [6], Remark 6.5)

$$
\gamma_{j}=\left(\frac{1-\sigma_{j}}{1+\sigma_{j}}\right)^{-1}\left|k_{j}\right|^{-1-N} \frac{\prod_{\ell=1}^{N}\left|1-k_{j} k_{\ell}\right|}{\prod_{\substack{\ell=1 \\ \ell \neq j}}^{N}\left|k_{j}-k_{\ell}\right|}, \quad 1 \leq j \leq N .
$$

We remark that these formulas can also be obtained by the inverse scattering transform (cf. e.g., [5], 15], 18, Section 3.6).

\section{ACKNOWLEDGMENTS}

I thank F. Gesztesy for bringing [4 to my attention and the referees for their valuable remarks.

\section{REFERENCES}

[1] R. Abraham, J.E. Marsden, and T. Ratiu, Manifolds, Tensor Analysis, and Applications, $2^{\text {nd }}$ edition, Springer, New York, 1983.

[2] M. Benammar and W.D. Evans, On the Friedrichs extension of semi-bounded difference operators, Math. Proc. Camb. Phil. Soc. 116, 167-177 (1994).

[3] W. Bulla, F. Gesztesy, H. Holden, and G. Teschl Algebro-Geometric Quasi-Periodic Finite-Gap Solutions of the Toda and Kac-van Moerbeke Hierarchies, Mem. Amer. Math. Soc. 135-641, (1998).

[4] P. Deift, L.C. Li, and C. Tomei, Toda flows with infinitely many variables, J. Func. Anal. 64, 358-402 (1985).

[5] H. Flaschka, On the Toda lattice. II, Progr. Theoret. Phys. 51, 703-716 (1974).

[6] F. Gesztesy and G. Teschl, Commutation methods for Jacobi operators, J. Diff. Eqs. 128, 252-299 (1996).

[7] F. Gesztesy and Z. Zhao, Critical and subcritical Jacobi operators defined as Friedrichs extensions, J. Diff. Eq. 103, 68-93 (1993).

[8] F. Gesztesy, H. Holden, B. Simon, and Z. Zhao, On the Toda and Kac-van Moerbeke systems, Trans. Amer. Math. Soc. 339, 849-868 (1993).

[9] M. Kac and P. van Moerbeke, On an explicitly soluble system of nonlinear differential equations, related to certain Toda lattices, Adv. Math. 16, 160-169 (1975).

[10] P. D. Lax Integrals of nonlinear equations of evolution and solitary waves, Comm. Pure and Appl. Math. 21, 467-490 (1968).

[11] R. M. Miura, Korteweg-de Vries equation and generalizations. I. A remarkable explicit nonlinear transformation, J. Math. Phys. 9, 1202-1204 (1968). 
[12] W. T. Patula, Growth, oscillation and comparison theorems for second order linear difference equations, SIAM J. Math. Anal. 6, 1272-1279 (1979).

[13] M. Reed and B. Simon, Methods of Modern Mathematical Physics II. Fourier Analysis, Self-Adjointness, Academic Press, San Diego, 1975.

[14] W. Renger, Toda soliton limits on general background, in Differential equations, asymptotic analysis, and mathematical physics, (ed. M. Demuth), 287-291, Math. Res. 100, Akademie Verlag, (1997).

[15] G. Teschl, Inverse scattering transform for the Toda hierarchy, Math. Nach. 202, 163171 (1999)

[16] G. Teschl, Spectral Deformations of Jacobi Operators, J. Reine und Angew. Math. 491, 16-47 (1997).

[17] G. Teschl, Oscillation theory and renormalized oscillation theory for Jacobi operators, J. Diff. Eqs. 129, 532-558 (1996).

[18] M. Toda, Theory of Nonlinear Lattices, $2^{\text {nd }}$ enl. edition, Springer, Berlin, 1989.

Institut für Reine und Angewandte Mathematik, RWTH Aachen, 52056 Aachen, GerMANY

Current address: Institut für Mathematik, Strudlhofgasse 4, 1090 Wien, Austria

E-mail address: Gerald.Teschl@univie.ac.at

URL: http://www.mat.univie.ac.at/〜gerald/ 